$\xi=\square$

\title{
Thermal properties of cubic zincblende thallium-phosphide from quasi-harmonic Debye model approximation
}

\author{
Salah Daoud * \\ Laboratory of Materials and Electronic Systems (LMSE), Faculty of Sciences and Technology, Mohamed Elbachir El Ibrahimi, \\ Bordj Bou Arreridj University, Bordj Bou Arreridj (34000), Algeria \\ *Corresponding author E-mail: salah_daoud07@yahoo.fr
}

\begin{abstract}
The thermal properties of cubic zincblende (B3) thallium - phosphide (TIP) compound under high pressure up to 12 GPa have been studied using the quasi-harmonic Debye model approximation. The relative unit cell volume, the isothermal bulk modulus, the first order derivative isothermal bulk modulus, the Debye temperature and the Grüneisen parameter are studied at zero-temperature and at standard ambient temperature $(298 \mathrm{~K})$ respectively. Our obtained values of the different previous quantities are in general in agreement compared to other theoretical data of the literature.
\end{abstract}

Keywords: Thermal Properties; Isothermal Bulk Modulus; Thallium- Phosphide Material; Quasi-Harmonic Debye Model Approximation.

\section{Introduction}

In view of the possibility for eventual technological applications, Thallium- phosphide material might be potential candidate, and it has attracted increasing theoretical research interest in the last few years [1]. To the best of our knowledge, this material and other materials of this family (TlAs and TlSb) have not been synthesized yet, and there are only a few theoretical works [1-9] on this materials are available.

Using plane-wave pseudopotential (PW-PP) approach in the framework of density-functional theory (DFT) within the local density approximation (LDA) and generalized gradient approximation (GGA), the phase transition, the structural parameters, the band structures, the total electronic density of states (DOS) and other thermophysical properties of cubic zincblende TIP and TlAs compounds have been studied by Ciftci et al. [2]. They found small values of phase transition pressure of these materials compared to other III-V materials.

Using ab initio Tight Binding-Linear Muffin Tin Orbital (TBMTO) within the local density approximation, Paliwal et al. [3] have reported, the total energy, structural stability, electronic band structure (BS), density of states (DOS) and several other physical quantities of some thallium pnictides $\mathrm{TlX}(\mathrm{Tl}=\mathrm{Sb}$, and $\mathrm{Bi})$ under ambient conditions in the CsCl-type structure. The $\mathrm{CsCl}$ structure has a body centre cubic structure with one formula unit per unit cell [3].

Saidi-Houat et al. [4] have used Ab initio self-consistent calculations in order to investigate the structural stability of the thalliumV compounds: TIN, TIP, TlAs, TlSb and TIBi. They found that the cubic zincblende (B3) structure of thallium phosphide (TIP) is the more stable phase compared to the other phases for the thallium phosphide compound. While the wurtzite (B4) structure is the more stable phase for TIN as found for other III-V nitrides.

Under hydrostatic pressure, the cubic zincblende (B3) phase of thallium phosphide compound disappears, and the cubic rock-salt
(B1) phase appears at a range pressure: $4.47 \mathrm{GPa}$ to $13.46 \mathrm{GPa}$ [1], [2], [5], [7], and [8].

In the present work, we have predicted the relative unit cell volume, the isothermal bulk modulus, the first order derivative isothermal bulk modulus, the Debye temperature and the Grüneisen parameter of Thallium- phosphide material at zero-temperature and at standard ambient temperature $(298 \mathrm{~K})$ in the range of pressure from 0 to $12 \mathrm{GPa}$. Our obtained results are discussed, analyzed and compared with other theoretical data of the literature.

\section{Computational methods}

In order to study the thermal properties of Thallium- phosphide material at zero-temperature and at standard ambient temperature $(298 \mathrm{~K})$ in the range of pressure from 0 to $12 \mathrm{GPa}$, the Debye model approximation [10] is applied; our calculations are implemented through the Gibbs code [11]. The Gibbs code is used to predict the thermodynamic properties from the minimization of Energy- Volume (E, V) data. The Energy-Volume (E, V) data can be obtained from several codes. The Energy-Volume (E, V) data used here are obtained from the Abinit code [12]. ABINIT code is a package allows finding the energy-volume $(\mathrm{E}, \mathrm{V})$ data, and some others physical quantities of systems made of electrons and nuclei. It is a common project of the University Catholique de Louvain, Corning Incorporated, the University de Liège, and other contributors. For more detail on the calculation of $(\mathrm{E}, \mathrm{V})$ data of this compound; please see our previous work [1], [5].

\section{Results and discussion}

The thermodynamic properties of TIP material in cubic zincblende phase at zero-temperature and at standard ambient temperature $(298 \mathrm{~K})$ and high pressures in the range of pressure from 0 to 12 $\mathrm{GPa}$ are studied and analyzed. Fig. 1 displays the variations of the relative unit cell volume $\mathrm{V}(\mathrm{p}) / \mathrm{V} 0$ as a function of pressure at 
zero-temperature and at $298 \mathrm{~K}$. Our results suggest that the relative unit cell volume decreases with increasing pressure, and at $298 \mathrm{~K}$, the fall in relative unit cell volume becomes relatively more important.

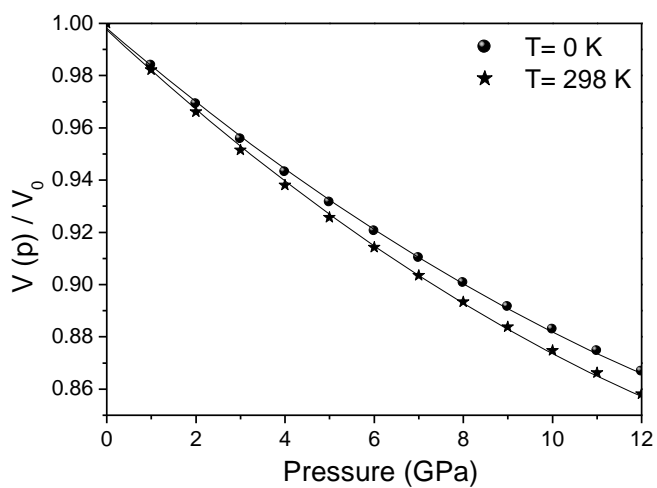

Fig. 1: Variations of the Relative Unit Cell Volume V (P) /V0 as A Function of Pressure at Zero-Temperature and At 298 K.

It is rather well known the pressure and temperature effects on bulk modulus of materials, because these two quantities play a very important role in the area of high-pressure and hightemperature science [13]. The isothermal bulk modulus $\mathrm{B}_{\mathrm{T}}$ is very important quantity, it can be determined as a function of pressure and temperature using the following expression [11]:

$$
\mathrm{B}_{\mathrm{T}}=-V(d p / d V)_{T}=V\left(d^{2} F / d V^{2}\right)_{T}
$$

Where: $\mathrm{F}$ is the vibrational Helmholtz free energy, $\mathrm{V}$ is the volume, $\mathrm{P}$ is the pressure, and $\mathrm{T}$ is the temperature.

Fig. 2 displays the pressure dependence of the isothermal bulk modulus at zero-temperature and at $298 \mathrm{~K}$. Our calculation results show that the pressure and the temperature play an opposite role on the isothermal bulk modulus, the isothermal bulk modulus increases with increasing pressure and decreases with temperature, At zero pressure and zero-temperature, our obtained value of the isothermal bulk modulus is $59.18 \mathrm{GPa}$; it is in excellent agreement with the value ( $59.19 \mathrm{GPa}$ ) obtained from elastic constants in our previous work [1]. Our obtained value of the isothermal bulk modulus is also in excellent agreement with the value (57.54 GPa) obtained by Ciftci et al. [2] using the local density approximation.

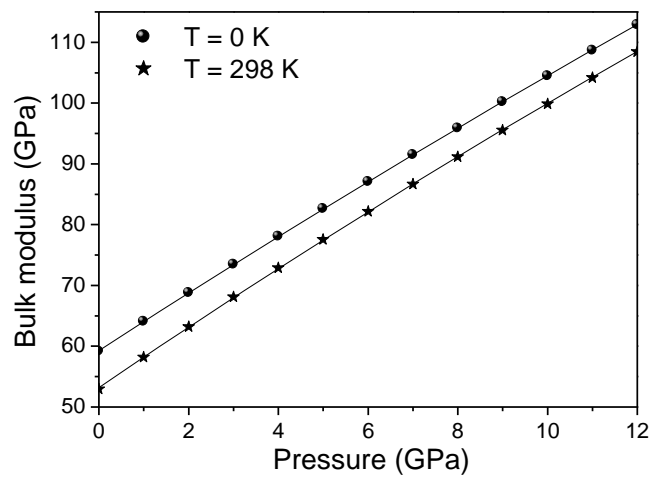

Fig. 2: Isothermal Bulk Modulus as a Function of Pressure at ZeroTemperature and At Standard Ambient Temperature.

At $\mathrm{T}=298 \mathrm{~K}$, an analytical relationship for the pressure dependence of $\mathrm{B}_{\mathrm{T}}$ is given by the following a quadratic fit:

$\mathrm{B}_{\mathrm{T}}=53.21+5.02 \mathrm{p}-3.47 \times 10^{-2} \mathrm{p}^{2}$

The variation of the first order derivative isothermal bulk modulus $\mathrm{B}^{\prime}$ as a function of pressure at zero-temperature and at $298 \mathrm{~K}$ is presented in Fig. 3. Our results suggest that $\mathrm{B}^{\prime}$ decreases with increasing pressure and increases with increasing temperature. At zero-pressure and zero-temperature the first order derivative isothermal bulk modulus takes the value 4.91, while for the shame pressure and $\mathrm{T}=298 \mathrm{~K}$, it takes the value 5.33.

At $\mathrm{P}=0$ and $\mathrm{T}=0 \mathrm{~K}$, our obtained value of $\mathrm{B}^{\prime}$ is in relatively higher than the value 4.425 obtained in our previous work [5] by using the Murnaghan equation of state (EOS), but it is in good agreement with the value (4.857 GPa) obtained by Ciftci et al. [2] using the Vienna ab initio simulation package (VASP) with the local density approximation (LDA).

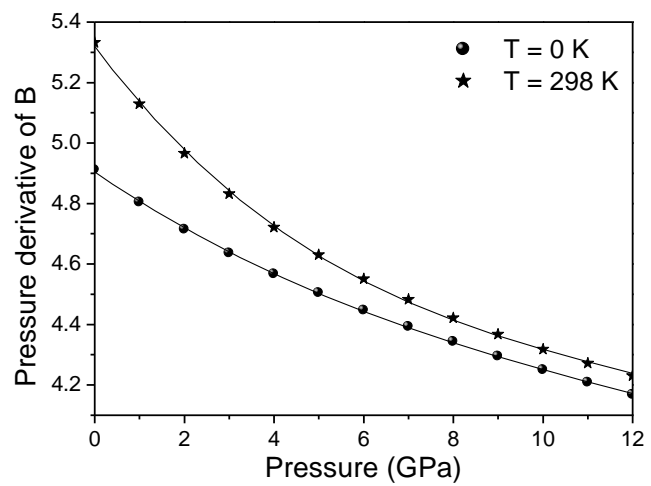

Fig. 3: Variations of First Order Derivative Isothermal Bulk Modulus B' as A Function of Pressure at Zero-Temperature and At $298 \mathrm{~K}$.

The Debye temperature $\theta_{\mathrm{D}}$ is one a fundamental thermophysical property of material; it is used to distinguish between high and low temperature regions [14]. In quasi-harmonic Debye model, $\theta_{\mathrm{D}}$ can be calculated using the following formula [11].

$\theta_{D}=\frac{}{k_{B}}\left[6 \pi^{2} V^{1 / 2}\right]^{1 / 3} f(\delta) \sqrt{\frac{B_{S}}{M}}$

Where: $\mathrm{M}$ is the mass of the unit cell, and $\mathrm{B}_{\mathrm{S}}$ is the adiabatic bulk modulus, it is approximated by the static compressibility

$$
\mathrm{B}_{\mathrm{S}} \approx \mathrm{B}_{\mathrm{V}}=\mathrm{V}\left(\frac{\partial^{2} \mathrm{E}(\mathrm{V})}{\partial \mathrm{V}^{2}}\right)
$$

And $\delta$ is the Poisson ratio, and it has been assumed that it is a constant.

$$
f(\delta)=\left\{3\left[2\left(\frac{2}{3} \frac{1+\delta}{1-2 \delta}\right)^{3 / 2}+\left(\frac{1}{3} \frac{1+\delta}{1-\delta}\right)^{3 / 2}\right]^{-1}\right\}^{1 / 3}
$$

Fig. 4 shows the calculated Debye temperature $\theta_{\mathrm{D}}$ as a function of pressure at zero-temperature and at $\mathrm{T}=298 \mathrm{~K}$. It can be seen from Fig. 4 that the Debye temperature increases quadratically with increasing pressure in the range 0 to $12 \mathrm{GPa}$. Our calculated value of the Debye temperature $\theta_{\mathrm{D}}$ at $\mathrm{P}=0$ and $\mathrm{T}=298 \mathrm{~K}$ is about 232.24 $\mathrm{K}$. It is relatively lower than the value $(259 \mathrm{~K})$ obtained in our work [5], and relatively higher than the value $(218.47 \mathrm{~K})$ obtained in our work [1], so it is localized between these two values.

It is shown that as the applied pressure increases from 0 to $12 \mathrm{GPa}$ $(3,6,9,12 \mathrm{GPa})$, the Debye temperature $\theta_{\mathrm{D}}$ increases very quickly $(11.5 \%, 21.45 \%, 30.15 \%, 37.83 \%)$. To the best of our knowledge, there are no experimental data or other theoretical results (except our results reported in Ref. [1]) at high pressure and standard ambient temperature $(298 \mathrm{~K})$ to be compared with our results of the Debye temperature $\theta_{\mathrm{D}}$. By fitting the pressure and Debye temperature $\theta_{\mathrm{D}}$ curve, the following quadratic function relationship at $\mathrm{T}=$ $298 \mathrm{~K}$ can be obtained as:

$\theta_{D}=233.19+9.20 \mathrm{p}-0.15 \times 10^{-2} \mathrm{p}^{2}$ 


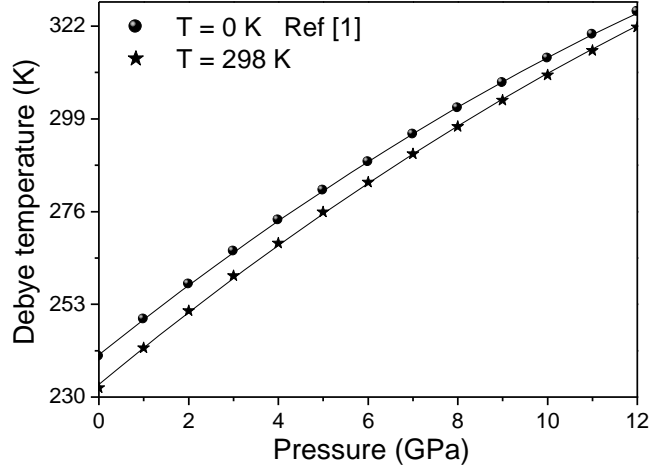

Fig. 4: Debye Temperature as a Function of Pressure at Zero-Temperature [1] and At Standard Ambient Temperature.

The Grüneisen parameter $\gamma$ is a quantity which describes the anharmonic effects in the vibrating lattice. At low-temperature, it was dominated by lower-frequency transverse modes [15]. It is usually given by the following expression [11]:

$\gamma=-\mathrm{d} \ln \theta(\mathrm{V}) / \mathrm{d} \ln \mathrm{V}$

Where: $\Theta(V)$ is the Debye temperature and V is the volume.

Fig. 5 displays the pressure dependence of the Grüneisen parameter $\gamma$ at zero-temperature and at $\mathrm{T}=298 \mathrm{~K}$; our calculation show that the temperature and pressure have opposite influences on $\gamma$. So contrarily to the Debye temperature $\theta_{\mathrm{D}}$, the Grüneisen parameter $\gamma$ decreases with increasing pressure and increases with temperature. At low temperature, the effect of pressure on $\gamma$ is more significant than that of temperature. At $\mathrm{T}=298 \mathrm{~K}$, it is shown that as the applied pressure increases from 0 to $12 \mathrm{GPa}(3,6,9,12$ $\mathrm{GPa})$, the Grüneisen parameter $\gamma$ decreases also quickly $(7.15 \%$, $11.63 \%, 15.06 \%, 17.94 \%)$.

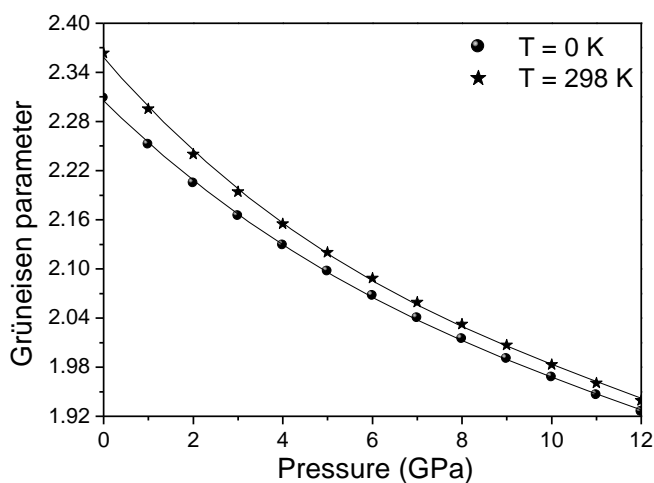

Fig. 5: Grüneisen Parameter as a Function of Pressure at ZeroTemperature [1] and At Standard Ambient Temperature.

At zero pressure and zero temperature, the obtained value of the Grüneisen parameter $\gamma$ is about 2.31; it is relatively higher than the value 1.734 estimated in our previous work [1], which is obtained by using the longitudinal $(\mathrm{vl})$ and transverse $\left(\mathrm{v}_{\mathrm{t}}\right)$ sound velocities. To the best of our knowledge, there is another data available in the literature on the Grüneisen parameter $\gamma$ for TIP material. So future experimental and other theoretical results are needed for the validating of our finding data.

\section{Conclusion}

Using the quasi-harmonic Debye model approximation, the thermal properties of (B3) thallium - phosphide (TIP) under high pressure up to $12 \mathrm{GPa}$ have been studied at zero-temperature and at standard ambient temperature $(298 \mathrm{~K})$. The relative unit cell volume, the isothermal bulk modulus, the first order derivative isothermal bulk modulus, the Debye temperature and the Grüneisen parameter are obtained, analyzed and compared to the other theoretical data of the literature. At $\mathrm{P}=0$, and $\mathrm{T}=0 \mathrm{~K}$, the majority of our obtained values of the different previous quantities are in good agreement compared to other results of the literature.

To the best of our knowledge, there is no data available in the literature at standard ambient temperature on the obtained quantities for this material.

\section{References}

[1] S. Daoud, "Mechanical and piezoelectric properties, sound velocity and Debye temperature of thallium-phosphide under pressure", International Journal of Advanced Research in Physical Science, Vol. 1, No. 6, (2014), pp. 1-11. www.arcjournals.org/pdfs/ijarps/v1i6/1.pdf.

[2] Y. O. Ciftci, K. Colakoglu, E. Deligöz, "A First Principles Studies on TIX (X=P, As)", Central European Journal of Physics, Vol. 6, No. 4, (2008), pp. 802-807. https://doi.org/10.2478/s11534-0080109-y.

[3] N. Paliwal, V. Srivastava, A. K. Srivastava, "Electronic Band Structure and Heat Capacity Calculation of Some TlX (X = Sb, Bi) Compounds", Advances in Materials Physics and Chemistry, Vol. 6 , No.3, (2016), pp. 47-53. https://doi.org/10.4236/ampc.2016.63005.

[4] N. Saidi-Houat, A. Zaoui, M. Ferhat, " Structural stability of thallium-V compounds", Journal of Physics: Condensed Matter, Vol. 19, No. 10, (2007), pp. 106221 (18pp). http://iopscience.iop. Org/09538984/19/10/106221/

[5] S. Daoud, N. Bioud, "Structural properties of (B3) TIP under pressure", International Journal of Physical Research. Vol. 2, No. 2, (2014), pp. 50- 55. https://doi.org/10.14419/ijpr.v2i2.3100.

[6] S. Daoud, N. Bioud, "Semi-Empirical Prediction of Physical Properties of (B3) TIP Compound", International Journal of Physical Research. Vol. 2, No. 2, (2014), pp. 72-77. http://dx.doi.org/10.14419/ ijpr.v2i2.3361.

[7] L. Shi, Y. Duan, L. Qin, " Structural Phase transition, electronic and elastic properties in $\mathrm{TlX}(\mathrm{X}=\mathrm{N}, \mathrm{P}, \mathrm{As})$ Compounds: pressureinduced effects", Computational Materials Science, Vol. 50, (2010), pp. 203-210. https://doi.org/10.1016/j.commatsci.2010.07.027.

[8] S. Singh, M. Sarwan, "High pressure phase transition and elastic behavior of TIX (X=N, P, as) semiconductors", Journal of Physics and Chemistry of Solids, Vol.74, (2013), pp. 487-495. https://doi.org/10.1016/j.jpcs.2012.11.016.

[9] M. Ferhat \& A. Zaoui, "Do all III-V compounds have the zincblende or wurtzite ground state structure?" Applied Physics Letters, Vol.88, No.16, (2006), pp. 161902-161904. https://doi.org/10.1063/1.2196050.

[10] M. A. Blanco, E. Francisco, V. Luaña, Gibbs: "Isothermal-isobaric thermodynamics of solids from energy curves using a quasiharmonic Debye model", Computer Physics Communications, Vol. 158, No.1, (2004), pp. 57-72. http://dx.doi.org/10.1016/j.comphy. 2003.12.001

[11] A. Otero-de-la-Roza and V. Luaña, "Gibbs2: A new version of the quasi-harmonic model code. II. Models for solid-state thermodynamics, features and implementation", Computer Physics Communications, Vol. 182, (2011), pp. 2232-2248. https://doi.org/10.1016/i.cpc.2011.05.009.

[12] The ABINIT code is a common project of the Université Catholique de Louvain, Corning Incorporated, and other contributors (URL http://www.abinit.org).

[13] X-W. Sun, Y.D. Chu, Z.J. Liu, T. Song, P. Guo, and Q.F. Chen, "Shell and breathing shell model calculations for isothermal bulk modulus in $\mathrm{MgO}$ at high pressures and temperatures", Materials Chemistry and Physics, Vol. 116, No.1, (2009), pp. 34-40. https://doi.org/10.1016/j.matchemphys.2009.02.021.

[14] D. P. Rai, M. P. Ghimire, R.K. Thapa, "A DFT Study of BeX (X = S, Se, Te) semiconductor: modified Becke Johnson (mBJ) Potential", Semiconductors, Vol. 48, No. 11, (2014), pp. 1447-1422. http://link.springer.com/article/10.1134/S1063782614110244. https://doi.org/10.1134/S1063782614110244.

[15] F. Kong, G. Jiang, "Phase transition, elastic, thermodynamic properties of zinc-blend BeSe from first-principles", Physica B, Vol. 404, No. 21, (2009), pp. 3935-3940. https://doi.org/10.1016/j.physb.2009.07.131. 